\title{
Traumatic subcutaneous emphysema after liposuction
}

\author{
Keun Tae Kim, \\ Hook Sun, \\ Eui Han Chung \\ Department of Plastic and \\ Reconstructive Surgery, Busan Paik \\ Hospital, Inje University School of \\ Medicine, Busan, Korea
}

Traumatic subcutaneous emphysema, which is the infiltration of air into subcutaneous tissues due to trauma, is caused by various factors such as chest and/or abdominal trauma, facial fractures, and barotrauma caused by mechanical ventilation. In this case report, a 32-year-old woman developed traumatic subcutaneous emphysema after undergoing abdominal liposuction at a local clinic. She was subsequently admitted to Busan Paik Hospital, and with early diagnosis and conservative treatment, she was discharged on the seventh day of hospitalization with no complications. However, because traumatic subcutaneous emphysema may accompany other injuries for various reasons, radiological examination and various tests should be performed to prevent serious complications and sequelae.

Keywords: Conservative treatment / Liposuctions / Traumatic subcutaneous emphysema

\section{INTRODUCTION}

Subcutaneous emphysema is the presence of air or gas in subcutaneous tissues, and it can be caused by trauma, surgery, assisted ventilation, medical or dental procedures, and numerous diseases.

Patients with subcutaneous emphysema have visible swelling in their heads, arms, and legs. Clinical symptoms can include crepitus on soft tissue palpation as well as chest pain and dyspnea. Chest X-rays can be used to make the diagnosis [1]. Herein, we report the clinical presentation and treatment of a 32-year-old female patient who arrived at Busan Paik Hospital with traumatic subcutaneous emphysema after liposuction at a local clinic.

\section{CASE REPORT}

The patient was a 32-year-old woman with no underlying dis-

Correspondence: Eui Han Chung

Department of Plastic and Reconstructive Surgery, Busan Paik Hospital, Inje

University School of Medicine, 75 Bokji-ro, Busanjin-gu, Busan 47392, Korea

E-mail: jeongyahn@gmail.com

Received November 19, 2018 / Revised February 14, 2019 / Accepted February 20, 2019 eases. The patient underwent previous liposuction under sedation at a local clinic. The procedure involved approximately 5-mm incisions made in the left and right medial umbilical regions. Liposuction did not include other areas, and the amount of fat suctioned was $1.5 \mathrm{~L}$, with a total operating time of 3 hours. Approximately 1 hour after the operation, the patient experienced muscle pain and swelling over her entire body. However, those who performed the procedure at the clinic indicated that the symptoms would improve over time. On the following day, systemic muscle pain worsened, which caused her to visit our emergency department.

The patient developed swelling over her entire body including her face and presented at our hospital's emergency department complaining of muscle pain and feeling hot (Fig. 1). During admission to the emergency department, the patient's vital signs were as follows: blood pressure, 120/80 mmHg; body temperature, $36.9^{\circ} \mathrm{C}$; pulse rate, 120 beats $/ \mathrm{min}$; and respiration rate, 22 breaths/min. Arterial blood gas analysis showed the following: $\mathrm{pH}$, 7.46; partial pressure of carbon dioxide, $33 \mathrm{mmHg}$; partial pressure of oxygen, $67 \mathrm{mmHg}$; standard bicarbonate, $23.5 \mathrm{mEq} /$ $\mathrm{L}$; and oxygen saturation, $94.0 \%$. Blood tests revealed that her white blood cell count was normal at 7,660/ $\mathrm{L}$, but her C-reac- 


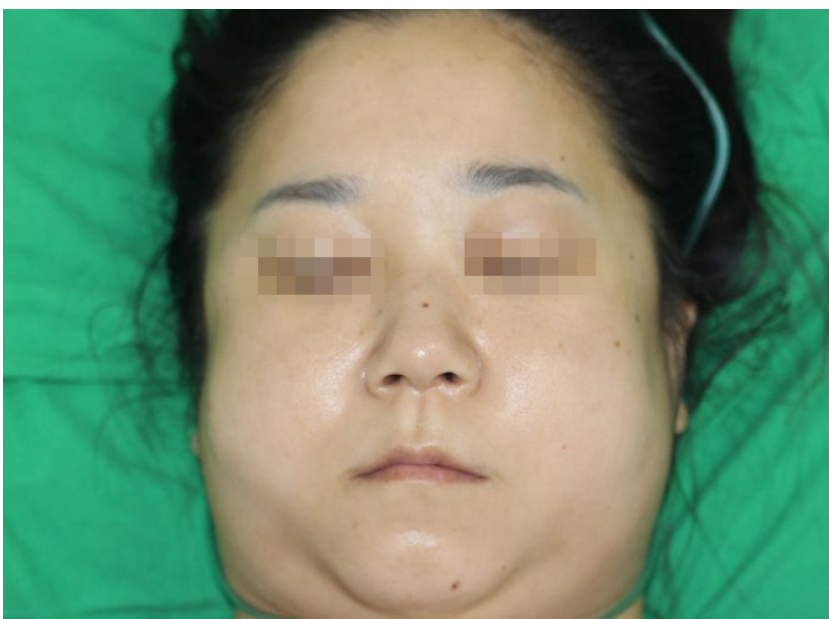

Fig. 1. Representative patient photograph showing extensive facial swelling after liposuction.

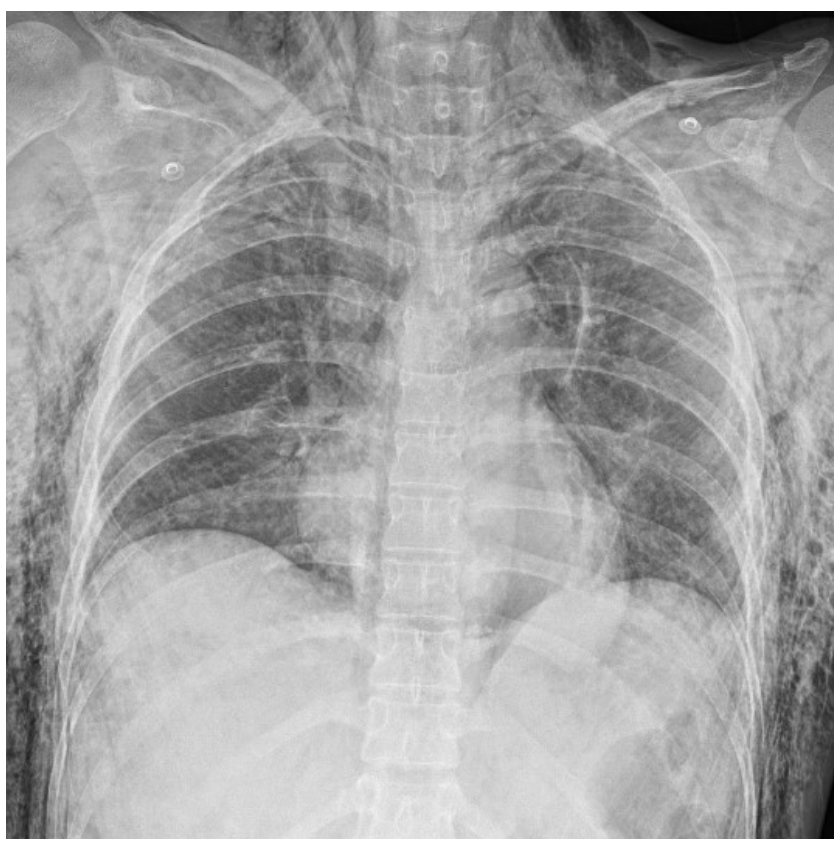

Fig. 2. Chest X-ray showing massive subcutaneous emphysema.

tive protein level was very high at $16.42 \mathrm{mg} / \mathrm{dL}$. Due to the high level of inflammatory markers, a further blood culture test was performed, but no specific bacteria were detected. The patient was alert, and her overall condition was fairly good. Physical examination demonstrated systemic edema including her face, as well as crepitus on palpation. On June 24, 2018, we performed chest radiography at our hospital and identified severe subcutaneous emphysema (Fig. 2). The patient also underwent chest computed tomography (CT), which revealed no fat embolisms or pulmonary edema, but a pneumomediastinum and bilateral pneumothoraces were observed. There were no specific findings on the chest CT image indicating any injury. More-
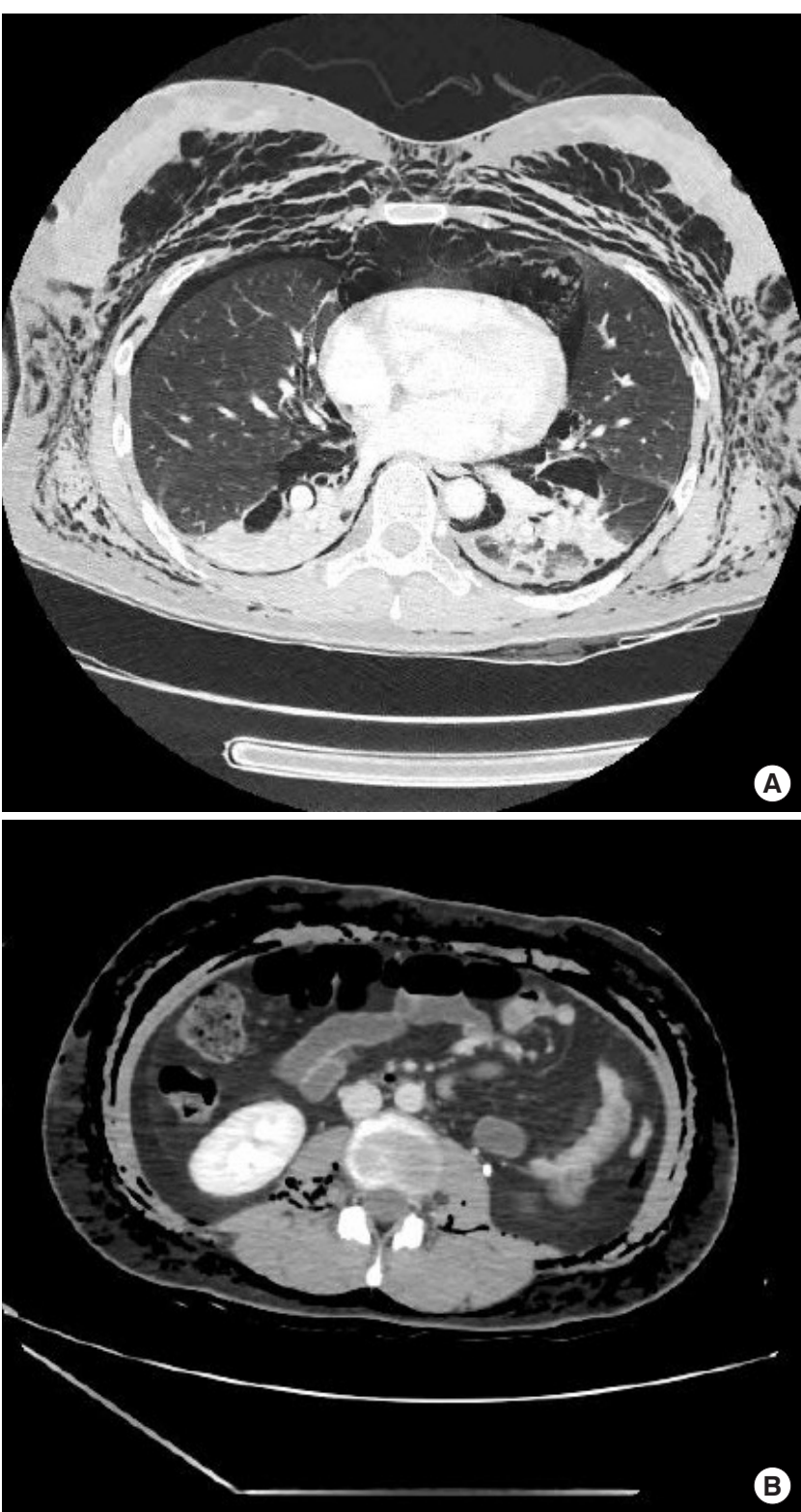

Fig. 3. (A) Chest computed tomography image showing massive subcutaneous emphysema, bilateral pneumothoraces, and pneumomediastinum. (B) Abdominal computed tomography image showing massive subcutaneous emphysema.

over, because the chest CT image did not appear to reveal a serious condition, our hospital managed the pneumothoraces and pneumomediastinum with only oxygen and antibiotics. In addition, abdominal CT showed no visceral perforation (Fig. 3). The patient was diagnosed with traumatic subcutaneous emphysema after liposuction and was treated as an inpatient in our department with preventive intravenous infusion of third-generation cephalosporin and 5-L oxygen therapy delivered via a mask for 6 days. The edema decreased by the 3rd day of hospitalization (Fig. 4), and the patient was discharged without complications on the 7th day (Fig. 5). 


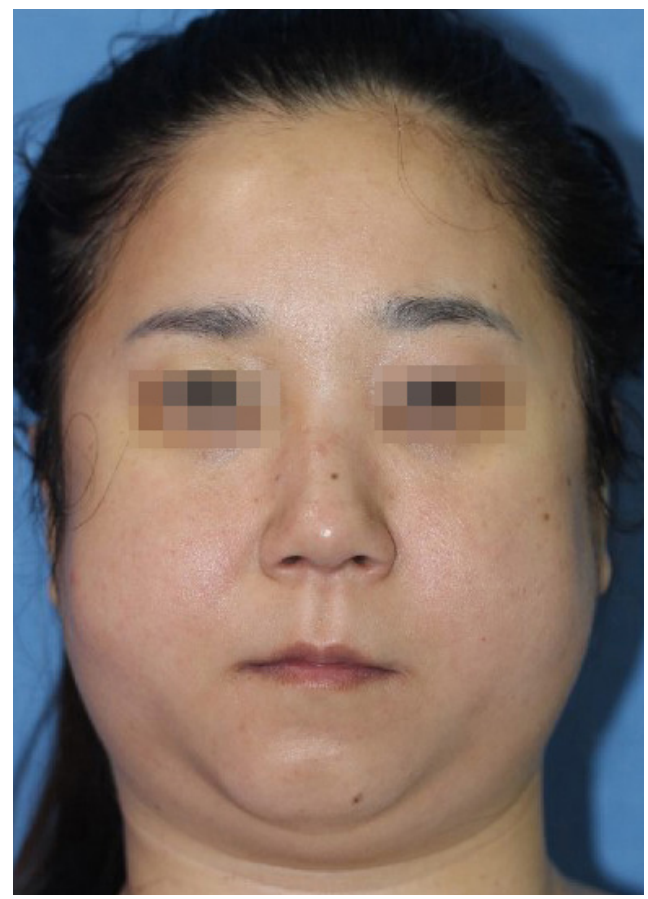

Fig. 4. Patient photograph on the 3rd day of hospitalization.

\section{DISCUSSION}

Traumatic subcutaneous emphysema is the infiltration of air into subcutaneous tissues as a result of trauma. Potential causes include thoracic or abdominal trauma, facial fractures, and pressure injuries due to assisted ventilation [2]. The clinical presentation of subcutaneous emphysema differs depending on the etiology. Patients typically show symptoms such as neck and chest pain as well as abdominal wall swelling, odynophagia, dyspnea, and tachypnea. On physical examination, crepitus is heard upon palpation [3].

Traumatic subcutaneous emphysema can result from various causes. Although the cannula must be inserted deep enough to be placed into the subcutaneous layer of the abdominal wall during liposuction, inserting it deeper may cause abdominal wall muscle or small bowel perforation injuries and subcutaneous emphysema [4]. When caused by a chest injury, it is typically accompanied by a pneumothorax and has the risk of death due to tracheal compression. In cases of trauma, often, there is injury to the facial skeleton; there may also be periorbital subcutaneous emphysema. There are various complications that may occur after liposuction, but among them, thromboembolism has a high mortality rate and is usually identifiable by CT. Moreover, it can be identified by clinical symptoms such as hypotension, bradycardia, no palpable pulses, and/or severe respiratory distress [5]. Cases of subcutaneous emphysema caused by liposuction, such as in the patient in this report, are uncom-

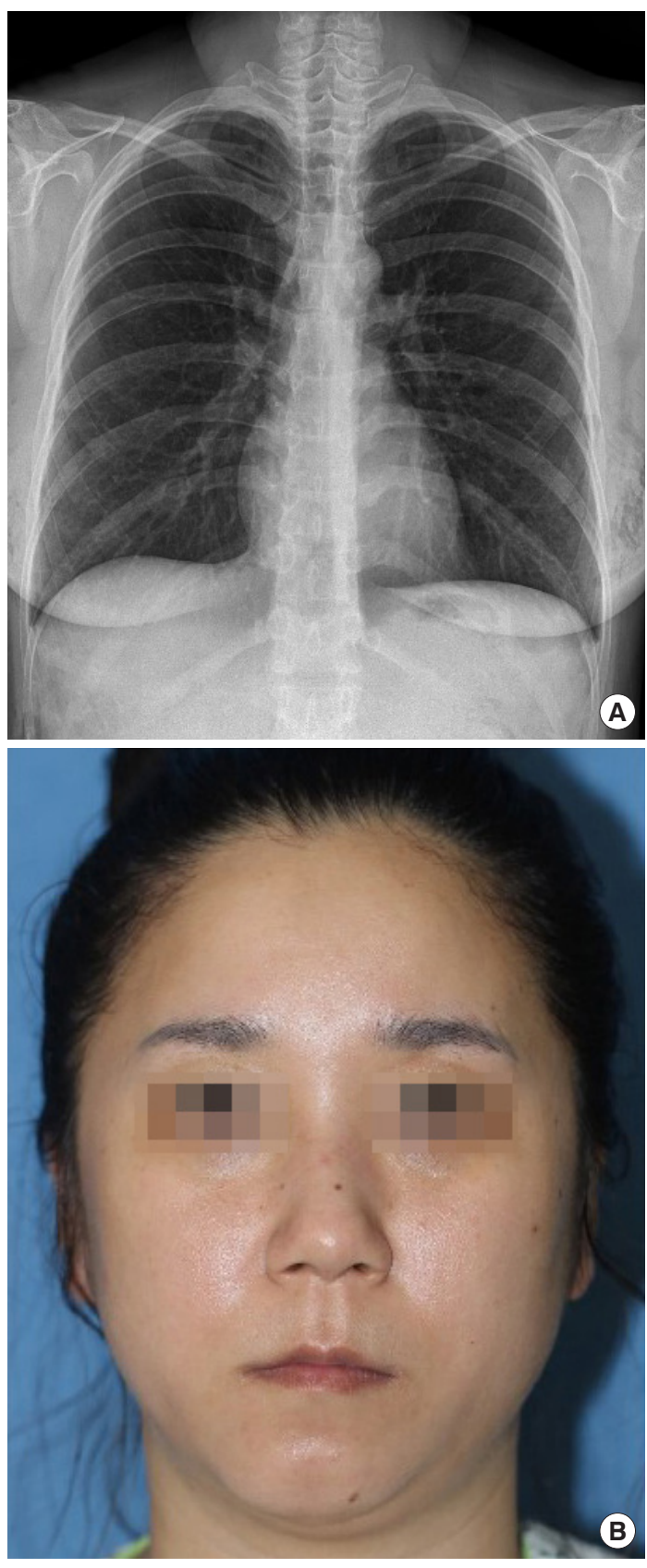

Fig. 5. (A) Chest posteroanterior X-ray showing resolution of subcutaneous emphysema. (B) Patient photograph on the 6th day after hospitalization.

mon. It is especially rare for such cases to show extensive subcutaneous emphysema throughout the body including the face. The mechanism of subcutaneous emphysema after liposuction was thought to involve the check valve in our case. Once air enters the subcutaneous space via the skin incision, it cannot escape due to the negative pressure differential and becomes trapped in the soft tissue, spreading along fascial planes and eventually developing into subcutaneous emphysema. As the second mechanism, aggressive liposuction with the cannula be- 
ing inserted deeper than the fat layer can cause a perforation, allowing air to enter the abdominal cavity. The air can flow along the space between the mediastinum and trachea to enter the chest cavity to cause a pneumomediastinum or pneumothorax. Moreover, the air can travel along the fascial surface of soft tissues. In such cases, introduction of air into the chest, neck, and facial area can cause a subcutaneous pneumothorax accompanied by crepitation; however, in the present case, there were no indications of parietal pleural injuries or abdominal wall injuries on CT images.

Fortunately, in the present case, the patient did not experience any injury to the chest cavity, abdominal cavity, or vital organs and did not have severe dyspnea or pain. However, unlike the present case, cases involving severe respiratory failure or exacerbation of systemic symptoms may require surgical treatment for decompression using a chest drain tube or an infraclavicular incision [6,7].

The severity and prognosis of traumatic subcutaneous emphysema differs depending on the etiology, but in most cases, with conservative treatment alone, symptoms are alleviated within 1 to 2 days and the patient recovers completely after 10 to 14 days [8]. There is a risk of infection in soft tissues, and fatal complications such as tracheal compression and mediastinitis can occur; thus, careful monitoring and broad-spectrum antibiotic treatment are required. In addition, bronchodilators can help with the clearance of airway secretions [9]. Supplying $100 \%$ oxygen can shorten the course of the disease because oxygen displaces subcutaneous air, thus increasing the rate of absorption into tissues.

To conclude, we reported the case of a patient who developed subcutaneous emphysema after liposuction. Even in cases of massive subcutaneous emphysema, such as in the present case, when there is no accompanying disease or injury to other areas, the patient can readily recover. However, because of the possibility of other injuries caused by liposuction, various examinations, including radiography, should be performed to confirm the diagnosis and patients should be carefully monitored to prevent severe complications and sequelae. Moreover, plastic surgeons must provide patients with sufficient information regarding possible complications associated with liposuction.

\section{NOTES}

\section{Conflict of interest}

No potential conflict of interest relevant to this article was reported.

\section{Ethical approval}

The study was approved by the Ethics Review Board of the Inje University Health Center (approval No. 19-0038) and performed in accordance with the principles of the Declaration of Helsinki. Written informed consent was obtained.

\section{Patient consent}

The patient provided written informed consent for the publication and the use of her images.

\section{ORCID}

Keun Tae Kim https://orcid.org/0000-0001-7753-739X

Hook Sun https://orcid.org/0000-0003-0104-2598

Eui Han Chung https://orcid.org/0000-0002-6581-4502

\section{REFERENCES}

1. Hopkins RL, Hamre M, Davis SH, Bonis SL, Frieberg EM. Spontaneous subcutaneous emphysema. Am J Emerg Med 1994;12:463-5.

2. Stringer DE, Dolwick MF, Steed DL. Subcutaneous emphysema after Le Fort I osteotomy: report of two cases. J Oral Surg 1979;37:115-6.

3. Stack BC Jr, Ridley MB. Spontaneous cervical emphysema in a child. Otolaryngol Head Neck Surg 1994;110:318-23.

4. Sharma D, Dalencourt G, Bitterly T, Benotti PN. Small intestinal perforation and necrotizing fasciitis after abdominal liposuction. Aesthetic Plast Surg 2006;30:712-6.

5. Uemura K, Kikuchi Y, Shintani-Ishida K, Nakajima M, Yoshida K. A fatal case of post-operative pulmonary thromboembolism with cosmetic liposuction. J Clin Forensic Med 2006;13:41-3.

6. Theodoros A, Athanasios K. Surgical treatment of subcutaneous emphysema. In which cases? J Surg Emerg Med 2017;1:12.

7. Lloyd MS, Jankowksi S. Treatment of life-threatening surgical emphysema with liposuction. Plast Reconstr Surg 2009;123: $77 \mathrm{e}-78 \mathrm{e}$.

8. Feinstone T. Infected subcutaneous emphysema: report of case. J Am Dent Assoc 1971;83:1309-11.

9. Steffey WR, Cohn AM. Spontaneous subcutaneous emphysema of the head, neck, and mediastinum. Arch Otolaryngol 1974;100:32-5. 